\title{
Environmental Regulation of Anther Dehiscence and Pollen Germination in Pecan
}

\author{
I.E. Yates \\ Russell Research Center, Agricultural Research Service, U.S. Department of Agriculture, Athens, \\ GA 30613 \\ Darrell Sparks \\ Department of Horticulture, University of Georgia, Athens, GA 30602-7273
}

Additional index words. Carya illinoinensis, pollination, fruit set, temperature, relative humidity, rehydration, pollen tube

\begin{abstract}
Detached anthers and pollen of pecan [Carya illinoinensis (Wangenh.) C. Koch] were exposed to a range of relative humidities (RH) and/or temperatures to assess the interaction of these environmental factors on pollen dispersal and germination. Dehiscence of anthers was evaluated at selected time intervals following exposure to $\approx 56 \%, 33 \%, 64 \%$, and $97 \%$ RH at 10, 21, 27, and 33C in a factorial experiment. Pollen release increased as RH decreased and temperature increased for detached anthers under laboratory conditions and for attached anthers under field conditions. Inhibition of anther dehiscence by high RH could be overcome or minimized with high temperatures and inhibition by low temperatures, in most cases, with low RH. Temperature effects on specific phases in the pollen germination process were evaluated in a sequence of experiments. First, pollen rehydration temperature was varied $(3,15,29,33$, and $42 \mathrm{C})$ and incubation temperature during tube development kept constant at $25 \mathrm{C}$. After 2 hours of rehydration, tube formation was maximized at $15 \mathrm{C}$ and tube length at 29C. Second, all pollen was rehydrated at a constant temperature (25C) and the incubation temperature during tube development varied $(3,15,29,33$, and $42 \mathrm{C})$. The temperature for maximum tube formation (15C) was different from that for maximum tube length (33C). Morphology of pollen tubes was normal under all rehydration conditions; but, during incubation for pollen tube development, tubes did not develop at $3 \mathrm{C}$ and developed abnormally at $42 \mathrm{C}$. The adverse effect on tube development at $3 \mathrm{C}$, but not at $42 \mathrm{C}$, could be reversed by transferring pollen to $25 \mathrm{C}$.
\end{abstract}

Fruit set in pecan, an anemophilous species with a heterdichogamous flowering habit (Sparks, 1986), depends on timely and adequate cross-pollination of receptive stigmatic flowers (Marquard, 1988; Sparks and Madden, 1985). Understanding the environmental factors that control pollen dispersal and viability is essential for predicting availability of pollen to effect fruit set and for establishing pollen collection protocol for mass supplemental pollination in commercial operations. Temperature and water are two of the most important environmental factors governing the periodicity of growth and development in plants (Kramer and Kozlowski, 1960; Slatyer, 1967).

Woodroof (1930) concluded from field data that anther dehiscence was governed predominantly by relative humidity $(\mathrm{RH})$, with temperature being of secondary importance. Periods of peak pollen release vary among cultivars and trees of the same cultivar, as well as within a tree and staminate inflorescence (Sparks, 1986; Yates and Sparks, 1992). Thus, pollen dispersal under field conditions can be erratic over time, even with similar temperatures and humidities. Woodroof's (1930) study was restricted to the environmental conditions and the developmental phase of the plant material existing during the time for data collection. Thus, interpolation to predict anther dehiscence is limited to conditions reported in the study. Woodroof (1930) expressed reservations that prevailing conditions were scored at the time of sampling and may not have represented the conditions during actual anther dehiscence.

Once pollen grains are released from the anther and land on an appropriate stigmatic surface, pollen germination is the next step leading ultimately to fertilization. Germination often is considered

Received for publication 31 July 1992. Accepted for publication 18 Dec. 1992. The technical assistance of Gloria Belvan, Leslie Browning, Joyce Lambert, and Donnie Maxey are gratefully acknowledged. Use of trade names does not imply endorsement of the products named nor criticism of similar ones not named. The cost of publishing this paper was defrayed in part by the payment of page charges. Under postal regulations, this paper therefore must hereby be marked advertisement solely to indicate this fact. a one-step process and reported as percent germination based on pollen grains with a tube of appropriate length. However, germination may be ordered into multiple steps, including rehydration, pollen tube formation, and tube elongation (Heslop-Harrison et al., 1975). Germination is used in this study to refer to these collective processes. Germination is analyzed as pollen tube formation and pollen tube elongation.

The time required for the sequential events in pecan pollen germination in vivo can be constructed with information obtained from several studies. Pecan pollen becomes rehydrated on the stigmatic surface within $\approx 1 \mathrm{~h}$ after pollination (Wetzstein and Sparks, 1989). Germ tubes equal or exceeding the grain diameter form within $\approx 3 \mathrm{~h}$ (Wetzstein and Sparks, 1989; Marquard, 1992). Germ tubes may penetrate the stigmatic surface as early as $2 \mathrm{~h}$ and elongate to beneath the junction of the stigma with the floral bracts within $7 \mathrm{~h}$ after pollination (Hinrichs and Ramming, 1973). Maximum percent pollen tube formation and elongation have been reported to occur in vivo and in vitro at 26 and 27C for 'Cheyenne' and 'Cape Fear' pecan, respectively (Marquard, 1992). However, the relationship of temperature with hydration and pollen tube development as sequential events was not analyzed. The objective of our study was to investigate the temperature and RH control of anther dehiscence and the range of temperatures over which pollen rehydration, pollen tube formation, and tube elongation can occur as progressive events in the germination process.

\section{Materials and Methods}

Temperature and RH control of anther dehiscence. Catkins, judged mature by criteria established previously (Yates and Sparks, 1992), were collected from 'Desirable' and 'Stuart' trees growing at the Univ. of Georgia Horticulture Farm, Watkinsville, for analysis of pollen release from detached anthers. Catkins were transported to the laboratory and anthers detached from the staminate inflorescence by pulling the catkin rachis gently between the 
fingers. Extraneous plant material, such as bracts, was removed by sifting the anthers through a sieve with $3-\mathrm{mm}^{2}$ openings. Any visibly damaged or abnormally small anthers were eliminated.

Preliminary experiments demonstrated that anther dehiscence under controlled environmental conditions in the laboratory varied with the conditions existing in the field when the catkins were collected. Thus, anthers were equilibrated to similar hydration states and temperature before experimentation. Anthers were wrapped in a moist paper towel and equilibrated overnight at 10C in a plastic bag.

Dehiscence of 'Desirable' and 'Stuart' anthers was analyzed in a split block $4 \times 4$ design replicated four times. The main plot was temperature and the subplot was RH. There were eight anthers per experimental unit. Anthers were placed in controlled environmental chambers at $10,21,27$, and $33 \mathrm{C}$. A low $\mathrm{RH}$ of $\approx 5 \%$ was established with solid $\mathrm{CaSO}_{4}$ at each temperature. Higher $\mathrm{RH}$ conditions were established with saturated salt solutions [33\% with $\mathrm{MgCl}_{2}, 64 \%$ with $\mathrm{NaNO}_{3}$, and $97 \%$ with $\mathrm{CuSO}_{4}$ (Young, 1967)]. Anthers were positioned on glass slides that were supported on plates mounted above the $\mathrm{CaSO}_{4}$ or salt solutions in glass desiccators.

Anthers were examined with a magnifying lens and scored as having dehisced when the anther suture split to expose the pollen. Time of observations varied because the response rate was different among temperatures. The cumulative number of dehisced anthers was scored hourly at $10 \mathrm{C}$ for the first $8 \mathrm{~h}$ and then at 12, 24, 48,72 , and $96 \mathrm{~h}$; at $21 \mathrm{C}$ on the same schedule as for 10C, except no scoring after $24 \mathrm{~h}$; hourly at $27 \mathrm{C}$ for the first $7 \mathrm{~h}$ and then at 10 , 12 , and $24 \mathrm{~h}$; and hourly at $33 \mathrm{C}$ for the first $6 \mathrm{~h}$ and then at 10 and $24 \mathrm{~h}$. Anther dehiscence was maximum or near maximum within $24 \mathrm{~h}$; hence, data for times after $24 \mathrm{~h}$ are not reported.

Results of pollen release from anthers detached from the tree and examined under laboratory conditions were corroborated with analyses of pollen release from anthers attached to trees under field conditions. Ten shoots on six separate 'Stuart' trees were marked when the majority of catkins were in either Stage III or Stage IV of maturity (Yates and Sparks, 1992). The most mature catkins were selected from each tree on succeeding days at 0900 HR. Catkins were placed in a laboratory oven at 32C. Monitoring pollen release of attached anthers in the field began the same day that pollen was released from detached anthers under laboratory conditions. Each shoot was examined for pollen release at hourly intervals and the temperature and RH were recorded. Minimal pollen was released on day 1 , reflecting the lack of uniform maturity of the catkins. Consequently, data on $\mathrm{RH}$, temperature, and percentage of shoots with pollen release was based on the average for each time interval for days 2,3 , and 4 .

Temperature control of pollen rehydration. Pollen was collected in Spring 1988 from 'Stuart' anthers. Pollen containing 8\% moisture was stored in liquid nitrogen. Pollen was removed from storage and rehydrated at $97 \% \mathrm{RH}$, by methods described for anther dehiscence, in controlled environmental chambers at either $3,15,29,33$, or 42C. Effects of rehydration for 10, 20, 30, 60, 120, and $240 \mathrm{~min}$ at each temperature on subsequent tube formation and elongation was determined by removing pollen from rehydration vessels and incubating pollen by standard protocol (Yates and Sparks, 1989). Briefly, the standard incubation protocol is to suspend $\approx 4000$ pollen grains in $0.2 \mathrm{ml}$ of medium in $12 \times 75-\mathrm{mm}$ test tubes and incubate pollen for $24 \mathrm{~h}$ at $25 \mathrm{C}$. After $24 \mathrm{~h}$, pollen grains were fixed with $2 \%$ glutaraldehyde containing $10 \%$ sucrose in $0.1 \mathrm{M} \mathrm{K}_{2} \mathrm{PO}_{4}(\mathrm{pH} 7.0)$ and stored at $5 \mathrm{C}$ until analyzed for pollen tube formation and elongation. Percent tube formation was determined for a minimum of 200 pollen grains in each of four test tubes and was based on the number of grains forming tubes of a length at least equal to the diameter of the grains. Pollen tube elongation for 30 grains in each of four test tubes was measured with a micrometer disk in a dissecting microscope.

Temperature control of pollen tube formation and elongation. Temperature effects on four aspects of pollen tube development was examined following rehydration under standard conditions. The aspects were: 1) the range of temperatures over which pollen tubes formed, 2) the rate of tube elongation, 3) the reversibility of temperature inhibition of pollen tube development, and 4) the threshold temperature causing deleterious effects on tube development. The pollen lot and other materials for these experiments were as described above for rehydration. Pollen was rehydrated by standard protocol (Yates and Sparks, 1989) at 97\% RH for $2 \mathrm{~h}$ at 25C. Pollen was then incubated for tube development as described above, except temperatures in controlled environmental chambers were maintained at 3, 15, 29, 33, and 42C. Percent tube formation and pollen tube length after $1,2,6,12$, and $24 \mathrm{~h}$ at each temperature were determined as described above.

The reversibility of adverse temperatures during tube development was analyzed by the standard protocol for rehydration and then incubating pollen for tube development at 3 and $42 \mathrm{C}$ for 1,2 , $4,6,12$, and $24 \mathrm{~h}$. At the end of the appropriate incubation time, pollen was then incubated at $25 \mathrm{C}$ for $24 \mathrm{~h}$. Tube development was compared to pollen incubated at $25 \mathrm{C}$ for $24 \mathrm{~h}$ immediately after rehydration.

The threshold temperature causing deleterious effects on pollen tube development was defined by incubating pollen grains at 33, 35 , and $37 \mathrm{C}$ for $2 \mathrm{~h}$ after rehydration. The percentage of pollen grains with normal and abnormal tube morphology was scored for each treatment.

Microscopy. Changes in pollen tube morphology associated with temperature of tube development were examined by scanning electron microscopy (SEM). Pollen grains in $\approx 20 \mu \mathrm{l}$ of incubation medium were placed on dialysis tubing laying on $1.5 \%$ agar to absorb the incubation medium, which required $\approx 30 \mathrm{~min}$. The pollen grains on the dialysis tubing were mounted on A1 stubs using double-sided tape, coated with gold-palladium, and examined at $15 \mathrm{kV}$ with a Philips 505 SEM (Division of North American Philips Corp., Mahwah, N.J.).

Statistical analyses. Data on the influence of temperature and/ or $\mathrm{RH}$ on anther dehiscence and pollen germination were subjected to regression analysis. Descriptive equations were derived for anther dehiscence, pollen tube formation and pollen tube length under all rehydration and incubation conditions. In cases of nonlinear models, the $r^{2}$ values were calculated as 1.0 minus the ratio of the residual sum of squares to the corrected total sum of squares (Ware et al., 1982).

\section{Results}

Temperature and RH control of anther dehiscence. Temperature and RH influenced dehiscence of anthers detached from 'Stuart' and 'Desirable' pecan trees (Fig. 1). Time required for initiation of anther dehiscence for 'Stuart' and 'Desirable' was equal under the more favorable conditions for anther dehiscence; i.e., high temperature and low RH (Fig. 1). However, 'Stuart' anthers consistently dehisced before 'Desirable' under less-favorable conditions. For example, anther dehiscence at $10 \mathrm{C}$ and $5 \% \mathrm{RH}$ was observed first at $2 \mathrm{~h}$ for 'Stuart' and $3 \mathrm{~h}$ for 'Desirable'.

The time required for $50 \%$ anther dehiscence decreased as the temperature increased and $\mathrm{RH}$ decreased with a strong interaction existing between these environmental factors (Fig. 2). At 64\% RH 
STUART

10C

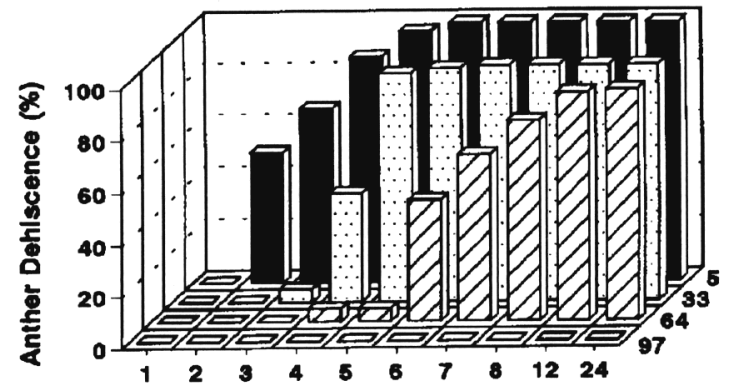

21C

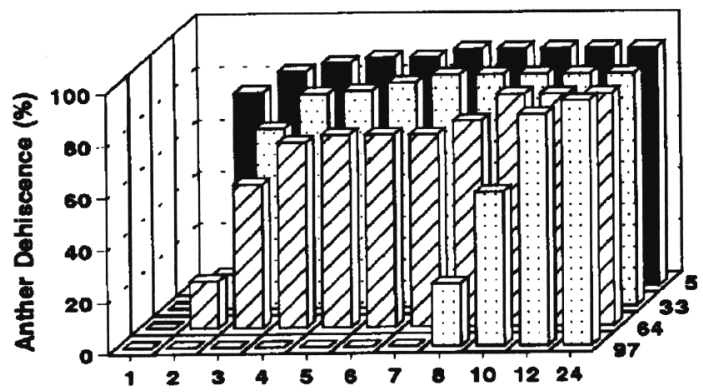

27C

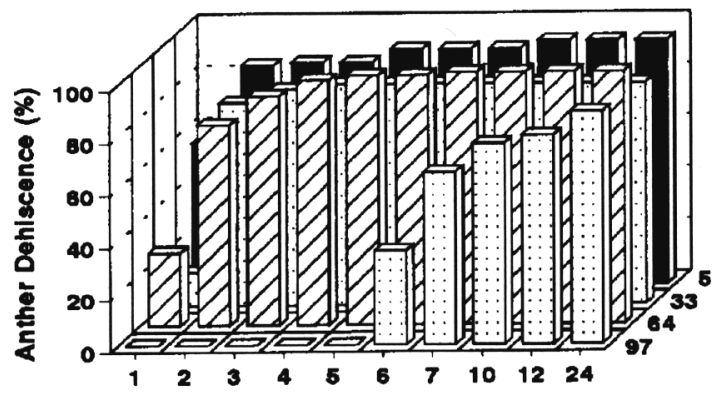

$33 C$

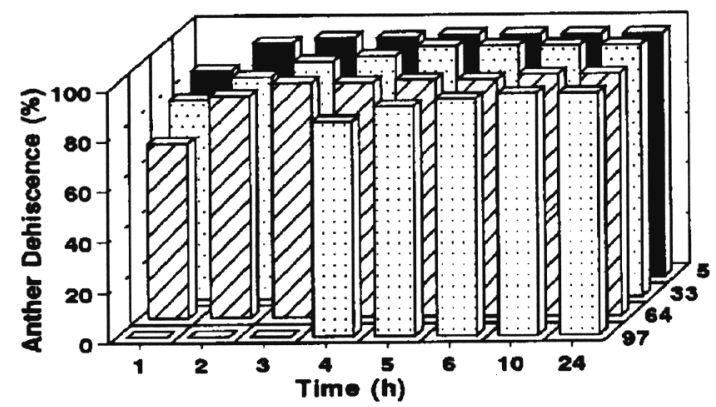

DESIRABLE

$10 \mathrm{C}$

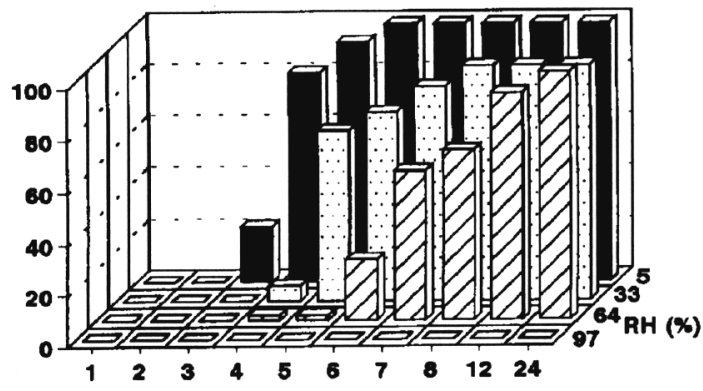

21C

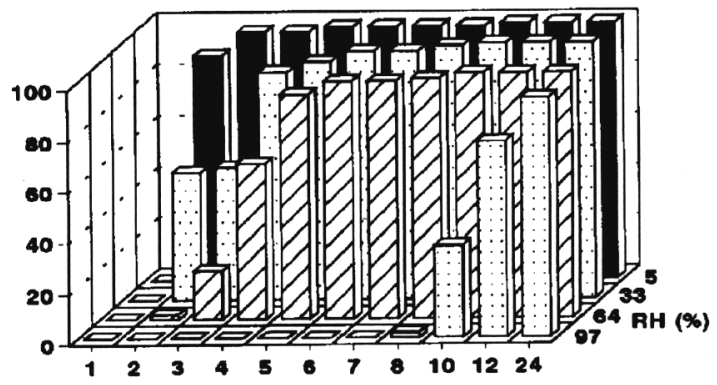

27C

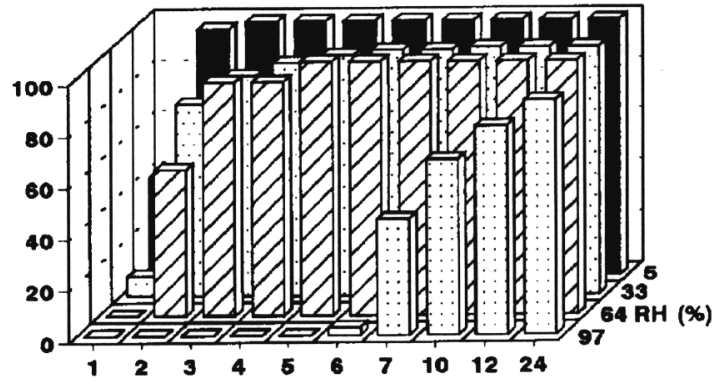

$33 C$

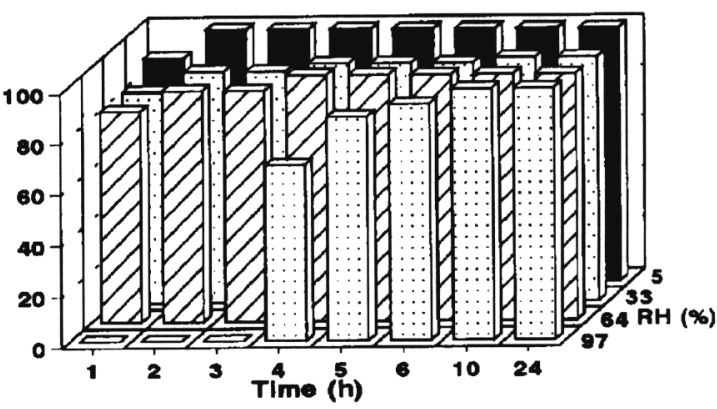

Fig. 1. 'Stuart' (left) and 'Desirable' (right) pecan pollen release from detached anthers under controlled RH and temperature conditions. The relationship of anther dehiscence $(\mathrm{Y})$ with time $(\mathrm{X})$ within a temperature and a RH followed Mitscherlich's plant growth model; $\mathrm{Y}=\mathrm{B}\left(1-\gamma \mathrm{e}^{-\mathrm{ax}}\right), r^{2}$ ranged from 0.879 to $0.999, P \leq 0.01$.

or less, the retarding effect of low $\mathrm{RH}$ was minimized with increasing temperature and was completely eliminated at 33C. However, at 97\% RH, anthers did not dehisce at 10C (Fig. 1), and higher temperatures only partially overcame the inhibition of extreme humidity (Fig. 2).

During dehiscence of anthers attached to 'Stuart' trees in the field, the $\mathrm{RH}$ decreased from $75 \%$ to $47 \%$ and temperature increased from $21 \mathrm{C}$ to $32 \mathrm{C}$ from 0800 to $1300 \mathrm{HR}$ (Fig. 3). The percentage of shoots with pollen release increased from $29 \%$ to $93 \%$ under these conditions. The largest hourly increase in anther dehiscence occurred from 1100 to $1200 \mathrm{HR}$, which was within $2 \mathrm{~h}$ after RH dropped to $59 \%$ and temperature increased to 27C (Fig. 3), as 


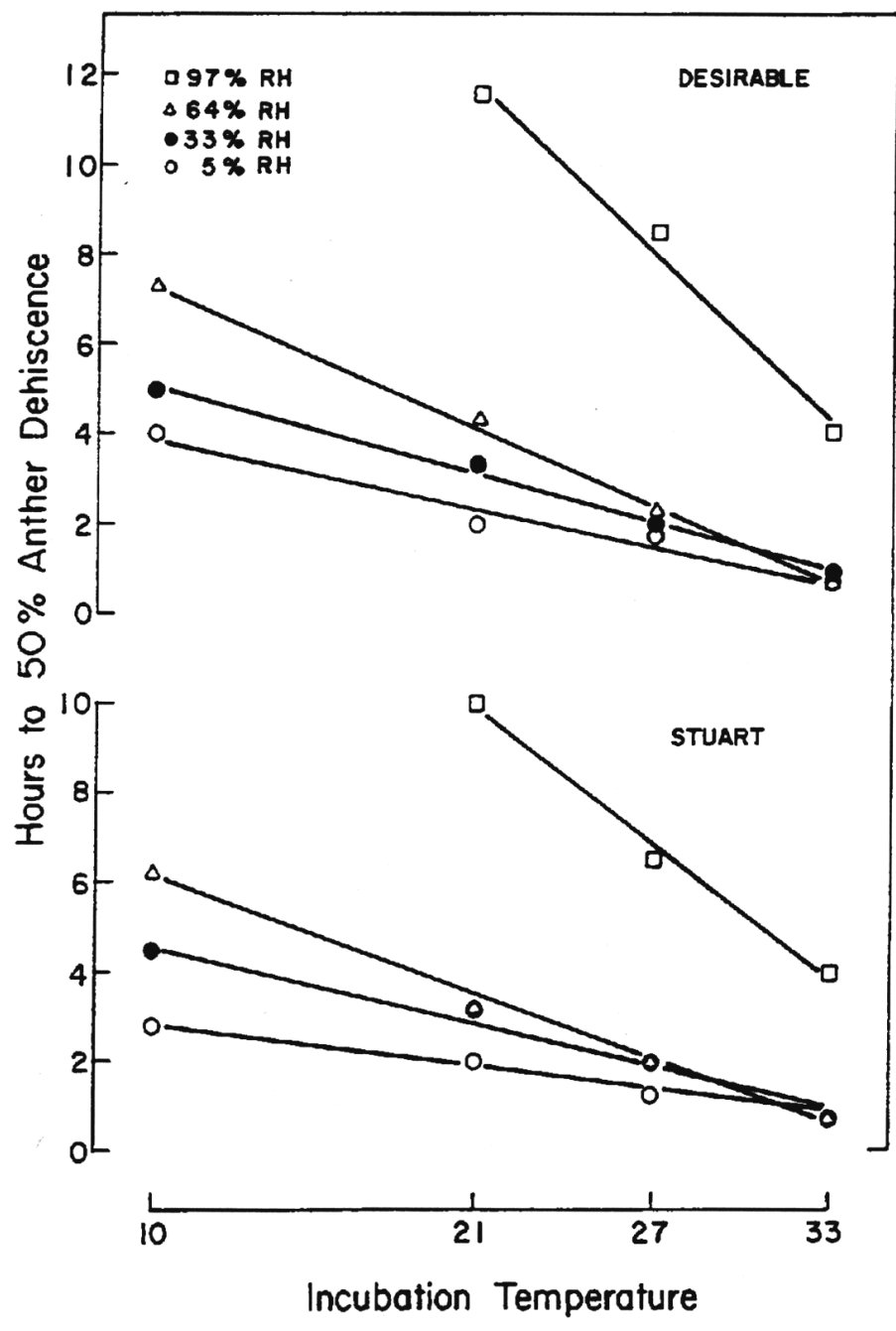

Fig. 2. Rate of anther dehiscence. The relationship of time to $50 \%$ anther dehiscence (y) and temperature (x) within a humidity was linear; $\mathrm{y}=\mathrm{a}-\mathrm{bx}, r^{2}$ for $5 \%, 33 \%$, $64 \%$, and $92 \%$ RH was $0.722,0.957,0.971$, and 0.977 for 'Stuart' and 0.935 , $0.975,0.976$, and 0.893 for 'Desirable', respectively, $P \leq 0.01$.

was observed for detached anthers at $27 \mathrm{C}$ and $64 \%$ RH (Fig. 1).

Temperature control during pollen rehydration. Even though both time and temperature of rehydration affected percentage of grains forming tubes (Fig. 4A) and pollen tube elongation (Fig. $4 \mathrm{~B})$, the response was different for these two measures of pollen tube development. Pollen tube formation was maximum at $15 \mathrm{C}$, regardless of rehydration time, and decreased either above or below 15C (Fig. 4A). Tube formation increased with rehydration time at all temperatures except 42C. Pollen grains rehydrated at $42 \mathrm{C}$ for 120 and $240 \mathrm{~min}$ did not form tubes. At all other temperatures, the effect of rehydration temperature on pollen tube formation was minimal following prolonged rehydration; i.e., $240 \mathrm{~min}$.

Pollen tube elongation was a direct function of rehydration temperature and was maximum at $42 \mathrm{C}$ at short rehydration times (<30 min) (Fig. 4B). The optimum rehydration temperature for tube elongation shifted from $42 \mathrm{C}$ down to $29 \mathrm{C}$ at longer rehydration times. Increasing rehydration time from 30 to $60 \mathrm{~min}$ at $42 \mathrm{C}$ suppressed pollen tube elongation, but not tube formation (Fig. 4 A vs. B). At other temperatures, tube elongation increased with rehydration time. However, as with pollen tube formation, there were only minimal temperature effects at prolonged rehydration times.

Temperature control during pollen tube formation and elongation. All pollen was rehydrated under common conditions $(25 \mathrm{C}$

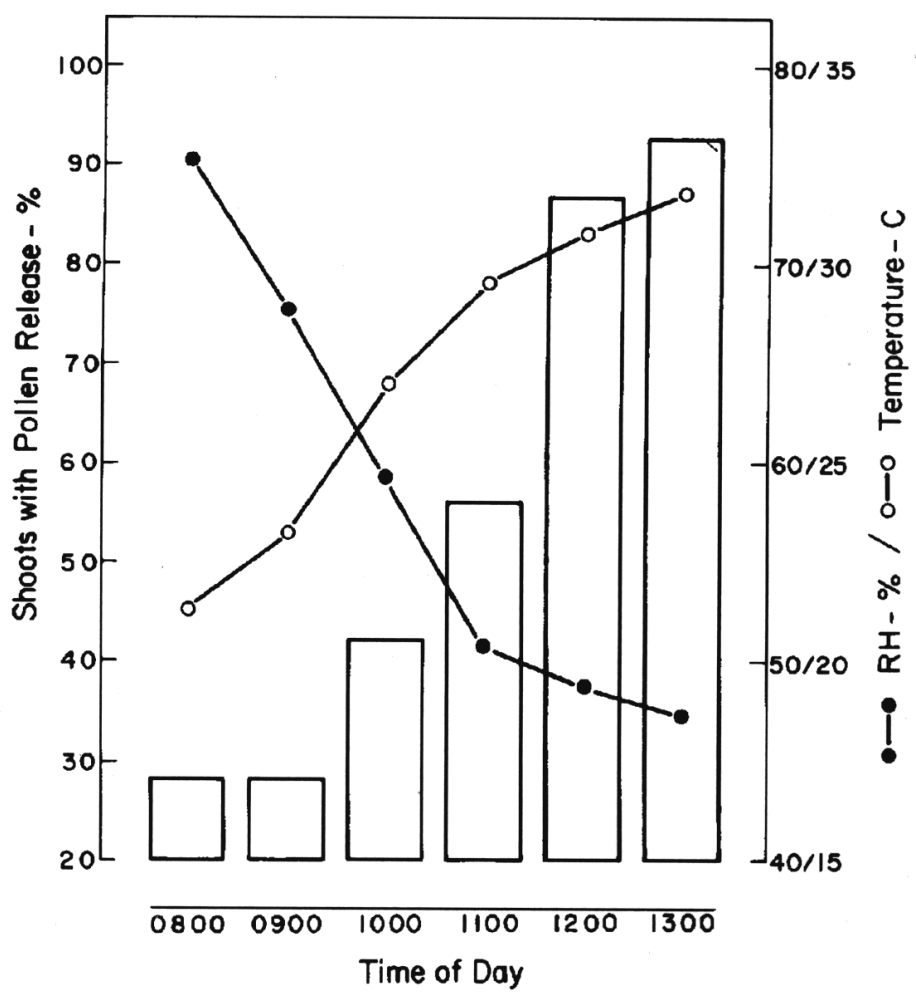

Fig. 3. Pollen release with time from anthers attached to 'Stuart' trees under existing field conditions of $\mathrm{RH}$ (percent) and temperature (C). Data for pollen release (bars) are the mean cumulative hourly percentage for 10 shoots on each of six 'Stuart' trees examined for 3 days during maximum pollen release.

and $97 \% \mathrm{RH}$ for $2 \mathrm{~h}$ ) and treated to selected temperatures during incubation in a liquid medium for pollen tube development. The temperature and length of the incubation period affected the percentage of pollen grains forming tubes, rate of pollen tube elongation, and morphology of pollen tubes (Figs. 5-7). Temperature was a critical factor regulating the length of pollen tubes at the end of a 24-h incubation period. In contrast, temperatures from 15 to $33 \mathrm{C}$ were not decisive in determining the percent tube formation. Tube formation after $1 \mathrm{~h}$ was $\leq 5 \%$, regardless of incubation temperature, but at $2 \mathrm{~h}$ became temperature-specific, with substantial formation being limited to 29 and 33C (Fig. 5A). The sequence of pollen tube formation over a period of $24 \mathrm{~h}$ at 29 and $33 \mathrm{C}$ is an initial rapid period of pollen tube formation followed by minimal increases, but for $15 \mathrm{C}$ is very staggered. Percentage of pollen tube formation at $15 \mathrm{C}$ for up to $12 \mathrm{~h}$ of incubation was less than that at either 29 or $33 \mathrm{C}$; but, after $24 \mathrm{~h}$, surpassed all other temperatures. Tube formation did not occur at $3 \mathrm{C}$ regardless of incubation time. Thus, pollen tube formation was specific for both incubation temperature and time.

Limited pollen tube elongation began with $2 \mathrm{~h}$ of incubation (Fig. 5B) and increased dramatically with increasing incubation time. Regardless of incubation time, tube elongation always increased with temperature up to a maximum at 33C. Prolonged incubation time did not override the depressing effect of temperatures lower than 33C. The time required for pollen tubes to elongate to $200 \mathrm{pm}$ or more was $2 \mathrm{~h}$ at $33 \mathrm{C}, 6 \mathrm{~h}$ at $29 \mathrm{C}$, and $12 \mathrm{~h}$ at $15 \mathrm{C}$.

Tube formation and elongation were greatly suppressed at $42 \mathrm{C}$ (Fig. 5). Grains forming tubes and pollen tube length at $42 \mathrm{C}$ were estimated as $5 \%$ and $50 \mathrm{pm}$, respectively, at all time periods. Estimation of the tubes formed at $42 \mathrm{C}$ was necessary because of 
A

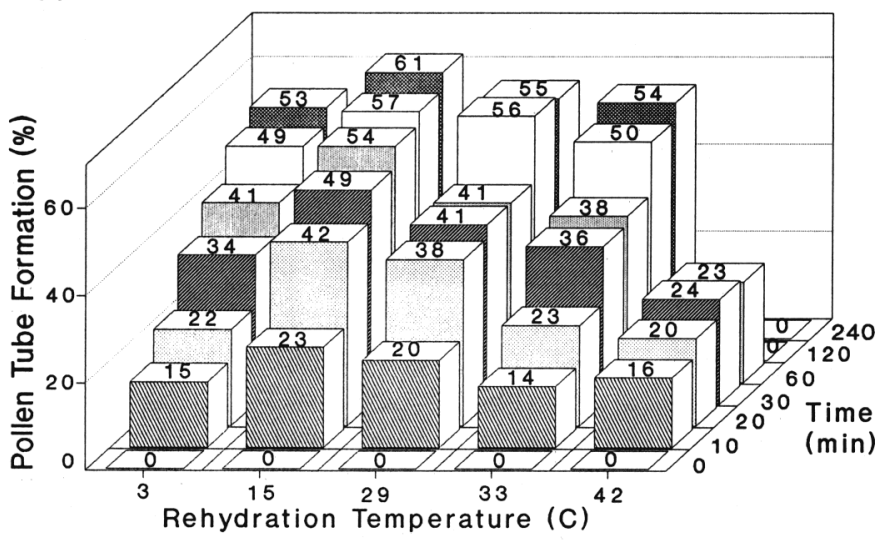

B

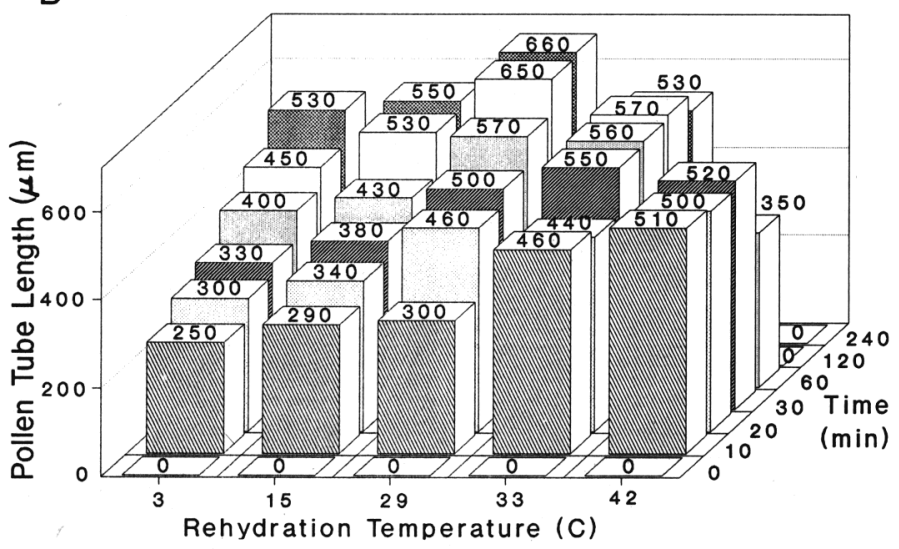

Fig. 4. Control of rehydration temperature on 'Stuart' pollen tube formation (A) and pollen tube length (B). Rehydration times examined were 0 to $240 \mathrm{~min}$ because pollen tube formation is maximized at $25 \mathrm{C}$ with $240 \mathrm{~min}$ rehydration (Yates and Sparks, 1989). The relationship of pollen tube formation (Y) with time (X) within a rehydration temperature followed Mitscherlich's plant growth model; $Y=B(1$ $\left.-\gamma e^{-a x}\right), r^{2}$ was $0.979,0.899,0.836$, and 0.925 , respectively. Pollen tube length with time within a rehydration temperature also followed Mitscherlich's curve and $r^{2}$ was $0.895,0.880,0.944$, and 0.991 for rehydration temperatures $3,15,29$, and $33 \mathrm{C}$, respectively, $P \leq 0.01$.

abnormal development. Some tubes appeared to be bulged (Fig. $6 \mathrm{~A}$ ) and others in various stages of eruption as if the structural integrity of the pollen tube tip were damaged (Fig. 6 B-D). Many times the erupted pollen tube contents were suspended in incubation medium without attachment to a pollen grain. Pollen tubes formed at 15, 29, and 33C appeared normal (Fig. 7).

As indicated above, pollen grains in incubation medium at $3 \mathrm{C}$ for up to $24 \mathrm{~h}$ did not develop tubes-the few tubes that did develop at $42 \mathrm{C}$ were damaged. Inhibition of pollen tube development at low (3C), but not high (42C), temperature could be overcome by transferring the pollen grains to incubation conditions at $25 \mathrm{C}$. The percent tube formation and elongation of pollen incubated in medium at $3 \mathrm{C}$ for $24 \mathrm{~h}$ and then at $25 \mathrm{C}$ for $24 \mathrm{~h}$ was equal to that of control pollen, which had never been treated to 3C. However, the effect of treating pollen at $42 \mathrm{C}$, even for only $1 \mathrm{~h}$, could not be reversed. Neither-percent tube formation nor elongation could be restored once the pollen grains had been treated at $42 \mathrm{C}$ for as short as $1 \mathrm{~h}$.

Pollen tubes formed at $33 \mathrm{C}$ were intact, but at $42 \mathrm{C}$ were damaged as described above. Pollen grains were incubated at $2 \mathrm{C}$ increments between $33 \mathrm{C}$ and $39 \mathrm{C}$ to assess more precisely the temperature causing tube damage. The highest temperature at which pollen tubes were intact was 33C (Table 1). Adverse effects
A

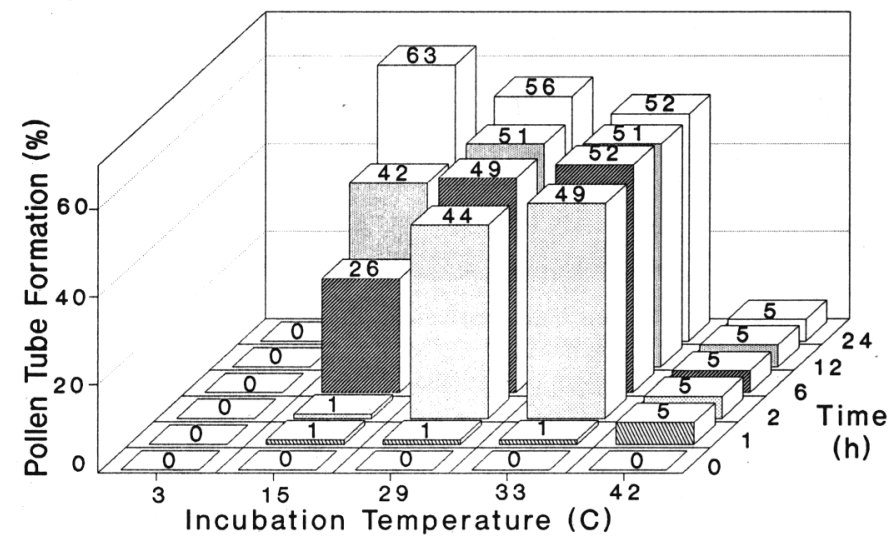

B

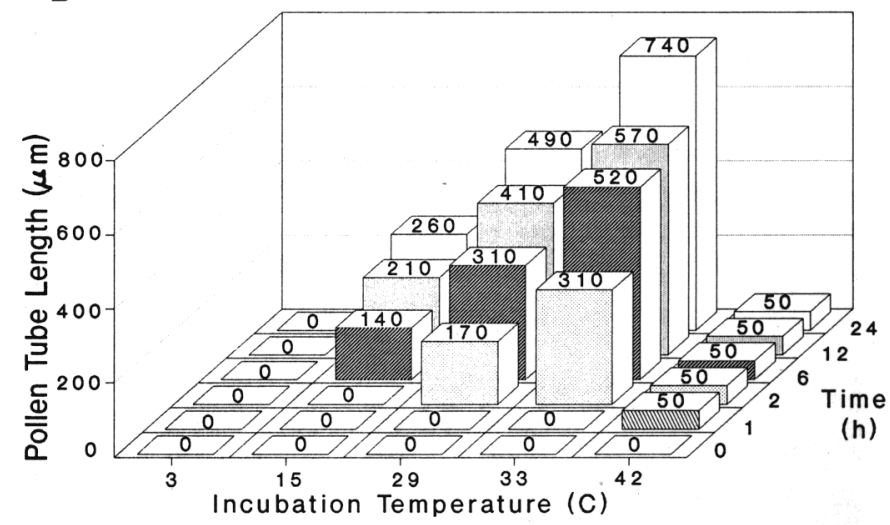

Fig. 5. Influence of incubation temperature on 'Stuart' pollen tube formation (A) and pollen tube length (B). Incubation times examined were 0 to $24 \mathrm{~h}$ because unpublished data demonstrated pollen tube formation was maximized at $25 \mathrm{C}$ with $24 \mathrm{~h}$ of incubation. The relationship of pollen tube formation ( $\mathrm{Y}$ ) with time (X) within incubation temperatures followed Mitscherlich's plant growth model; $\mathrm{Y}=\mathrm{B}\left(1-\gamma \mathrm{e}^{-\mathrm{ax}}\right), r^{2}$ was $0.976,0.928$, and 0.968 for temperatures 15,29 , and $33 \mathrm{C}$, respectively. Pollen tube length with time within an incubation temperature likewise followed Mitscherlich's equation and $r^{2}$ was $0.977,0.956$, and 0.889 for temperatures 15,29 , and $33 \mathrm{C}$, respectively, $P \leq 0.01$.

became evident at $35 \mathrm{C}$, where the percentage of abnormal tubes was $18 \%$ (Table 1). At 39C, the percent tube formation was $<10 \%$, and no normal tubes were formed.

\section{Discussion}

Pecans are grown as a commercial crop in the United States under extremes of humidity from the humid Southeast to the arid West. Under extremely low humidity (5\% RH, Fig. 2), ambient temperature would have only a negligible effect on pollen release. In humid climates, the typical $\mathrm{RH}$ and temperature range during the pollen release period is $\approx 40 \%$ to $70 \%$ and 21 to $31 \mathrm{C}$, respectively (Woodroof, 1930). Thus, the RH in humid climates would substantially retard pollen release at temperatures significantly

Table 1. Temperature-related progression of abnormal tube development in 'Stuart' pecan pollen.

\begin{tabular}{lcc}
\hline \hline Temperature $\left({ }^{\circ} \mathrm{C}\right)$ & Tuber formation $(\%)$ & Abnormal tubes $(\%)$ \\
\hline 33 & 54 & 0 \\
35 & 54 & 18 \\
37 & 38 & 33 \\
39 & $<10$ & 100 \\
\hline
\end{tabular}




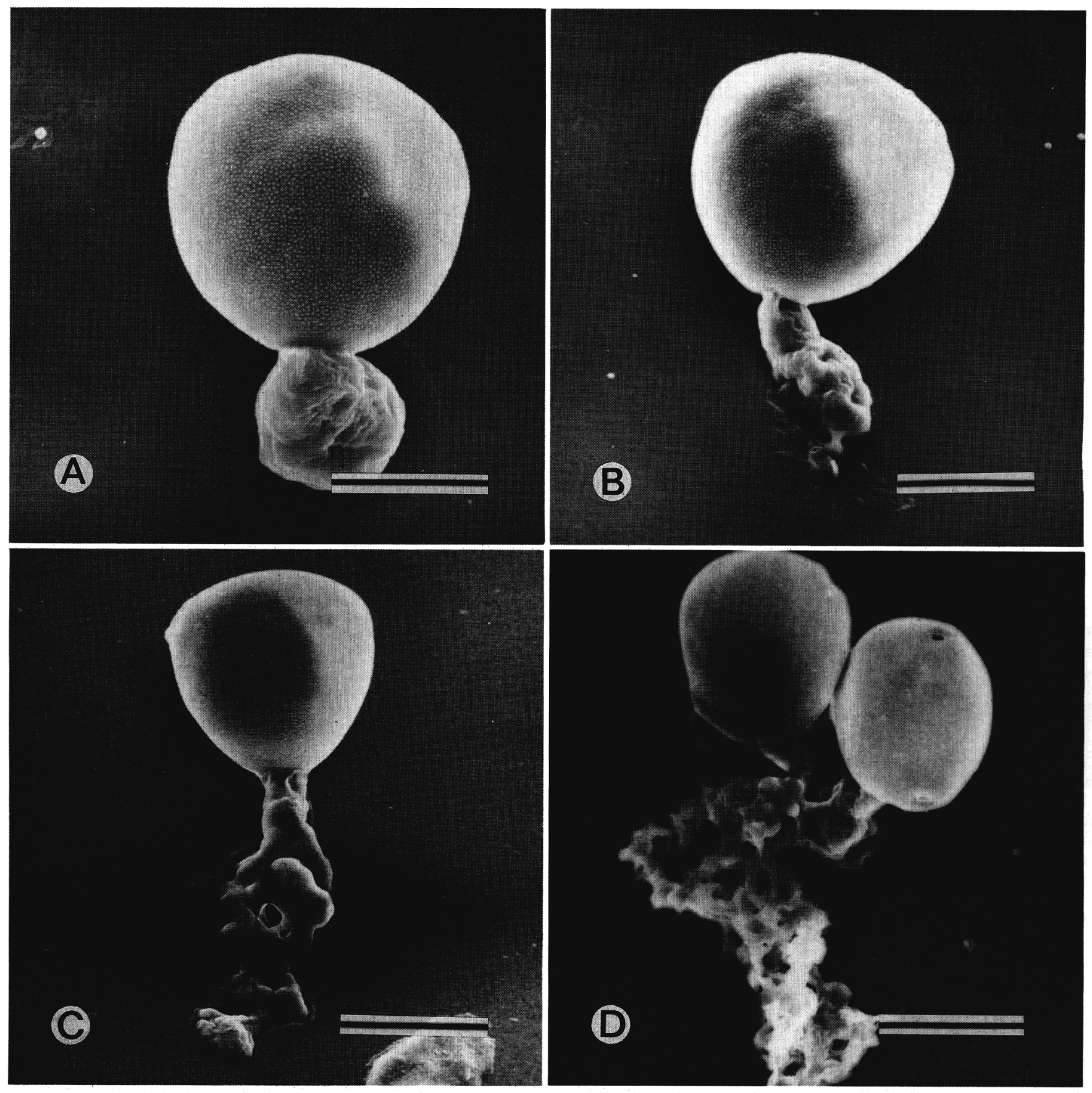

Fig. 6. Scanning electron micrographs of progressive deterioration of 'Stuart' pollen tubes at $42 \mathrm{C}$ for $2 \mathrm{~h}$ in incubation medium. Pollen tube development ranged from a bulge (A) to less than the diameter of a pollen grain with minor tip disruption $(\mathbf{B})$, to greater than the diameter of pollen grain with extensive tube disruption $(\mathbf{C}$ and D). (A and B) Bar $=20 \mu \mathrm{m}$. (C and D) Bar $=30 \mu \mathrm{m}$.

below 21C (Fig. 2). Our study concurs with that of Woodroof (1930) in that pollen release is inhibited by increasing humidity. High humidity inhibition is commonly observed under field conditions, as pollen shed does not occur during rainy periods. However, once rains cease and $\mathrm{RH}$ falls, pollen release is massive. Inhibition of anther dehiscence by high $\mathrm{RH}(97 \%$, Fig. 2) is an apparent survival mechanism associated with the mode of pecan pollen dispersal, which is by wind. Pollen release during rains would limit pollen distribution both by increase in pollen weight and by lack of uniform distribution by winds.

In theory, the high humidity inhibition of pollen release under field conditions can be partially overcome by high temperatures (Fig. 2). However, during the pollen release period, temperatures required for overcoming high humidity inhibition, i.e., $21 \mathrm{C}$ or above (Fig. 2), do not occur during high humidity or else are not sustained long enough to remove inhibition (Woodroof, 1930). Regulation of anther dehiscence in the field is not practical, but the results do have implications for pollen collection for supplemental pollination in commercial operations. To optimize the quantity of pollen released from anthers, catkins should be placed at $33 \mathrm{C}$ and as low humidity as possible.

The sequence of environmental conditions found to be optimum in vitro for pecan anther dehiscence, pollen hydration, tube formation, and tube elongation in the current study mimic field conditions during the spring, when pollen is shed and pistillate flowers are pollinated. Under field conditions, pollen shed in- 


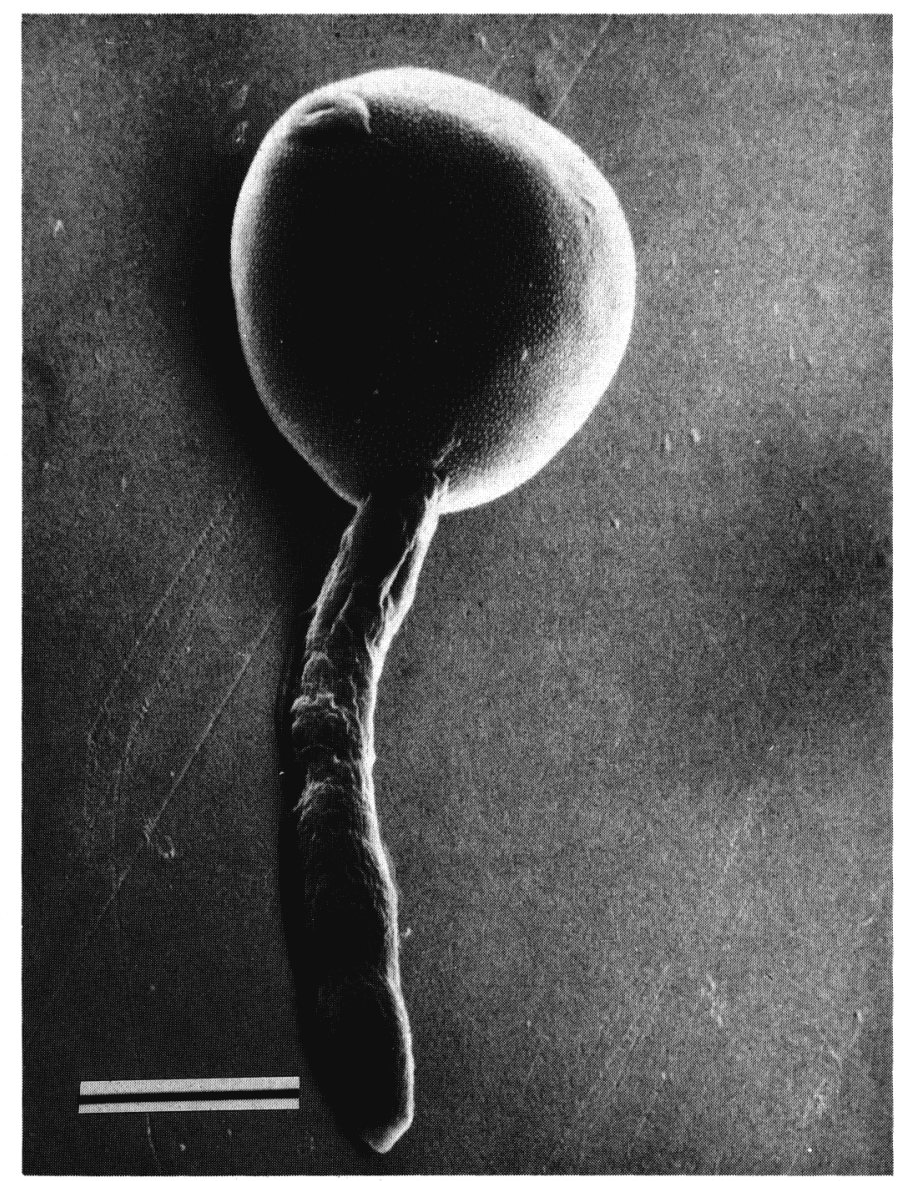

Fig. 7. Scanning electron micrograph of normal development of 'Stuart' pollen tube at $29 \mathrm{C}$ for $2 \mathrm{~h}$ in incubation medium. Pollen tube is intact and elongated to about twice the diameter of the pollen grain. $\mathrm{Bar}=30 \mu \mathrm{m}$.

creased as RH decreased and temperature increased and was maximized at $1200 \mathrm{HR}$, which was within $2 \mathrm{~h}$ after RH dropped to $59 \%$ and temperature increased to $27 \mathrm{C}$.

Once pollen grains are released from the anthers, the next order of events is pollen rehydration on the stigmatic surface, which occurs within $\approx 1 \mathrm{~h}$ after pollination (Wetzstein and Sparks, 1989). Within the conditions tested, maximum pollen tube formation after rehydration for $1 \mathrm{~h}$ was at $15 \mathrm{C}$, with the longest tubes formed at 29C. Again, this information has implications in artificial pollination, either for breeding or commercial supplemental pollination purposes. Pollen should be distributed in the field in the early morning while temperatures are relatively low for pollen rehydration to maximize the number of germinating pollen grains.

Pollen rehydration is followed by formation of germ tubes, which requires $\approx 3 \mathrm{~h}$ on the stigmatic surface for tubes to develop to a length that equals or exceeds grain diameter (Marquard, 1992; Wetzstein and Sparks, 1989). Within the conditions tested, pollen tube formation within this period was maximum at 29C, which is above the temperature that is optimal for rehydration. Likewise, in the field, day temperatures normally would increase from the time of rehydration to tube formation.

Pollen tube formation is followed by elongation. Indirect analyses of pollen tube growth in pistillate flowers under field conditions indicate that pollen tubes elongate to beneath the junction of the bracts with the stigmatic surface within $7 \mathrm{~h}$ after pollination (Hinrichs and Ramming, 1973). Under laboratory conditions in our study, the temperature for maximum pollen tube elongation (33C) was higher than that for maximum tube formation (29C). Again, shifting to higher temperatures for pollen tube elongation is analogous to elevation of day temperatures under field conditions as pollen tubes grow through the stigmatic tissue following pollination.

The optimum rehydration, tube formation, and elongation temperatures derived for 'Stuart' pollen may not be the optimum temperatures for all pecan cultivars. For 'Stuart' pollen, rehydration and incubation temperatures for maximum percent tube formation differed from that for maximum tube elongation. Maximum tube formation occurred with pollen rehydrated at $15 \mathrm{C}$, but subjected to 29 to $33 \mathrm{C}$ during tube formation itself. Then, the longest pollen tubes were formed at 33C. In contrast, Marquard (1992) reported that 'Cape Fear' pollen germination percentage and tube length in vitro were maximal at $27 \mathrm{C}$, with maximum tube length in vivo for 'Cheyenne' pollen at a slightly lower temperature. The results of the current study and that of Marquard (1992) are similar in that both demonstrate pecan pollen has a sharp decline in heat tolerance at temperatures higher than optimal for the given pollen. Differences defining the exact temperature at which the heat intolerance becomes a factor may be due to either genotypic differences (i.e., 'Stuart' vs. 'Cape Fear'), analytical technique (i.e., liquid vs. agarsolidified medium), temperature interactions for tube formation vs. elongation, environmental conditions during pollen development (not reported for either study), or a combination of these factors. Optimum germination temperatures may not be the same for a given cultivar in different growing seasons. Polito et al. (1991) have demonstrated that the temperature to which walnut pollen is exposed during development dictates the optimum temperature for germination. Likewise, the optimum temperature for pollen germination of a given pecan cultivar may vary also from year to year or site to site, contingent on prevailing temperatures during microsporogenesis.

Temperature during either rehydration or incubation affected pollen tube formation and elongation in vitro and could have implications for growth of pollen tubes on pistillate flowers in vivo. Low temperatures were more restrictive during incubation than rehydration (Figs. 4 and 5), indicating pollen tube formation and elongation may be more temperature-dependent than rehydration. Tube formation commenced more quickly and elongation, proceeded more rapidly at higher than lower temperatures (Fig. $5 \mathrm{~A}$ and B). Additionally, elongation was inhibited by low temperatures, even though a large percentage of pollen grains formed tubes. In contrast to tube formation, high temperatures must be maintained for maximum elongation. Thus, prolonged cool weather could restrict both pollen tube growth rate and final length by either inhibiting or delaying fertilization of the egg apparatus beyond a productive interval. Abnormally high fruit abortions possibly may occur in years with prolonged cool weather during the pollination period.

Pollen tube morphology was normal at all rehydration temperatures, but abnormal at incubation temperatures $\geq 35 \mathrm{C}$. These results imply that extremes of high, as well as low temperatures during pollination could reduce fruit set. However, the temperature of a stigmatic flower still attached to the tree may be ameliorated by plant influences so that temperatures high enough to interfere with pollination may not exist in vivo.

In conclusion, temperature optima under laboratory conditions for the processes of anther dehiscence, pollen rehydration, and pollen tube development follow a pattern in accord with the order of succession of temperatures under field conditions. Further, the influence of elevated temperatures on anther dehiscence has commercial applications for collection of pollen to use in supplemental pollination of large pecan acreages. The impact of extreme temperatures on pollen germination may have implications for fruit set in years with unseasonable temperatures during pollination. 


\section{Literature Cited}

Heslop-Harrison, J., R.B. Knox, Y. Heslop-Harrison, and O. Mattsson. 1975. Pollen-wall proteins: Emission and role in incompatibility responses, p 189-202. In: J.G. Duckett and P.A. Racey (eds.). The biology of the male gamete. Academic, London.

Hinrichs, H.A. and D.W. Ramming. 1973. Flowering and nut development of pecans. Proc. Okla. Pecan Growers Assn. p. 21-28.

Kramer, P.J. and T.T. Kozlowski. 1960. Physiology of trees. McGrawHill, New York.

Marquard, R.D. 1988. Outcrossing rates in pecan and the potential for increased yields. J. Amer. Soc. Hort. Sci. 113:84-88.

Marquard, R.D. 1992. Pollen tube growth in Carya and temporal influence of pollen deposition on fertilization success in pecan. J. Amer. Soc. Hort. Sci. 117:328-331.

Polito, V.S., S.A. Weinbaum, and T.T. Muraoka. 1991. Adaptive responses of walnut pollen germination to temperature during pollen development. J. Amer. Soc. Hort. Sci. 116:552-554.
Slatyer, R.O. 1967. Plant-water relationships. Academic, New York. Sparks, D. 1986. Pecan, p. 323-339. In: S.P. Monselise (ed.). CRC Handbook of fruit set and development. CRC Press, Boca Raton, Fla. Sparks, D. and G.D. Madden. 1985. Pistillate flower and fruit abortion in pecan as a function of cultivar, time, and pollination. J. Amer. Soc. Hort. Sci. 110:219-223.

Ware, G.O., K. Ohki, and L.C. Moon. 1982. The Mitscherlich plant growth model for detetminmg critical nutrient deficiency levels. Agron. J. 74:88-91.

Wetzstein, H.Y. and D. Sparks. 1989. Stigma-pollen interactions in pecan. J. Amer. Soc. Hort. Sci. 114:355-359.

Woodroof, J.G. 1930. Studies of the staminate inflorescence and pollen of Hicoria pecan. J. Agr. Res. 40:1059-1104.

Yates, I.E. and D. Sparks. 1989. Hydration and temperature influence in vitro getmination of pecan pollen. J. Amer. Soc. Hort. Sci. 114:599-605. Yates, I.E. and D. Sparks. 1992. External morphological characteristics for histogenesis in pecan anthers. J. Amer. Soc. Hort. Sci. 117:181-189. Young, J.F. 1967. Humidity control in the laboratory using salt solutions-a review. J. Applied Chem. 17:241-245. 